\title{
NUMERICAL MODELING OF TWO-PHASE FLOW WITH CONTACT DISCONTINUITIES
}

\author{
RONALD A. REMMERSWAAL ${ }^{*}$ AND ARTHUR E.P. VELDMAN ${ }^{\dagger}$ \\ Johann Bernoulli Institute, University of Groningen \\ Nijenborgh 9, 9747 AG Groningen, The Netherlands \\ *r.a.remmerswaal@rug.nl ${ }^{\dagger}$ a.e.p.veldman@rug.nl
}

Key words: two-phase flow, contact discontinuity, breaking waves

\begin{abstract}
When simulating high Reynolds number two-phase flow, boundary layers develop at the interface, which are much thinner compared to the capillary length-scales that are of interest. Resolving such an interface layer is expensive and therefore it is often not resolved in a simulation.

Numerically such an underresolved interface layer results in a velocity discontinuity tangential to the interface. We propose to include such tangential velocity discontinuities in our numerical model. This results in a sharp two-fluid model for two-phase flow, where only the interface-normal component of the velocity field is smooth. This condition is implicitly enforced via a new jump condition on the pressure gradient, which we discretize using a multidimensional variant of the ghost fluid method [6].

Results are shown of breaking waves [2] as well as (breaking) waves impacting a solid wall [3] where we compare to state-of-the-art methods [3,4]. We show that our proposed method is able to accurately simulate high Reynolds number two-phase flow without the need for resolving, or artificially thickening, of the interface layer.
\end{abstract}

\section{INTRODUCTION}

Numerical simulations can facilitate in the understanding of the physics underlying the obeserved variability in impact pressures during breaking wave impacts [3]. One of the mechanisms deemed responsible for pressure variability is the development of free surface instabilities at the wave crest. Numerical investigation of free surface instabilities on a breaking wave crest requires resolving the capillary length scales, amongst which the Kelvin-Helmholtz length scale ${ }^{1}$ will be most restrictive. Due to viscous effects, an interface layer will form at the fluid interface, whose thickness ${ }^{2} \lambda^{\mathrm{BL}}$ is estimated to be significantly smaller than the capillary length scale that we are interested in resolving

$$
\frac{\lambda^{\mathrm{BL}}}{\lambda^{\mathrm{KH}}} \approx \frac{\sqrt{\left|\llbracket u_{\tau} \rrbracket\right|}}{40},
$$

where $\llbracket u_{\tau} \rrbracket$ is the tangential velocity difference accross the interface. To that end we propose to model the interface layer by letting the velocity field be discontinuous in the interface tangential direction.

In section 2 we describe the continuous model which will be discretized in section 3 . Results are shown

\footnotetext{
${ }^{1}$ Here $\lambda^{\mathrm{KH}}$ denotes the fastest growing wavelength according to linearized potential flow theory.

${ }^{2}$ This length scale is estimated using the Blasius boundary layer approximation for a single phase flow.
} 
in section 4 followed by concluding remarks in section 5 .

\section{MATHEMATICAL MODEL}

We consider a $d$-dimensional domain $\Omega \subset \mathbb{R}^{d}$, which is separated by a time-dependent interface $I(t)$ resulting in a liquid domain $\Omega^{l}(t)$ and a gaseous domain $\Omega^{g}(t)$. Since the Navier-Stokes equations are equal for the liquid and gas phase, we state them for the $\pi$-phase, where we consider $\pi \in\{l, g\}$.

At the interface we define the jump of some function $\alpha$ in the following way

$$
\llbracket \alpha \rrbracket(\mathbf{x})=\alpha^{g}(\mathbf{x})-\alpha^{l}(\mathbf{x})=\lim _{s \rightarrow 0^{+}} \alpha(\mathbf{x}+s \eta)-\lim _{s \rightarrow 0^{-}} \alpha(\mathbf{x}+s \eta), \quad \mathbf{x} \in I,
$$

where $\eta$ denotes the interface normal pointing into the gas phase. Similarly we define the sum as

$$
\{\alpha\}\}=\alpha^{g}+\alpha^{l} .
$$

\subsection{Two-fluid model}

We model the two-phase flow using a two-fluid model, where each of the phases is modeled by the incompressible Navier-Stokes equations, which we write here in conservative form on some arbitrary and fixed control yolume $\omega \subset \Omega$

$$
\frac{d}{d t} \int_{\omega^{\pi}} \rho^{\pi} d V+\int_{\partial \omega^{\pi} \backslash I} \rho^{\pi} u_{\eta}^{\pi} d S=0
$$
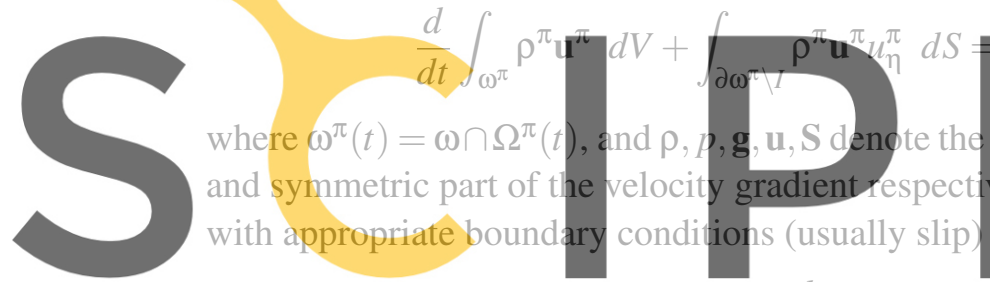

$$
\frac{d}{d t} \mathbf{x}=\mathbf{u}^{\pi}(\mathbf{x}, t), \quad \forall \mathbf{x} \in I(t),
$$

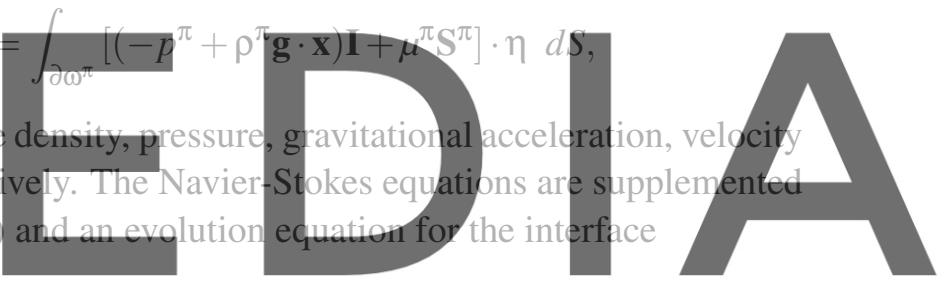

(2)

Register for free at https//www.scipediti.com to download the version without the watermark where the interface motion may thus be modeled by either of the two velocities. The following velocity

jump condition then uniquely determines the interface evolution

$$
\llbracket u_{\eta} \rrbracket=0,
$$

where $u_{\eta}=\mathbf{u} \cdot \eta$. Furthermore we impose the Young-Laplace equation on the interface to model the effect of surface tension (we omit the jump in the diffusive stresses)

$$
\llbracket p \rrbracket=-\sigma \kappa,
$$

where the interface mean curvature is denoted by $\kappa$ and $\sigma$ denotes the surface energy coefficient.

We choose to combine the two mass conservation equations (1) in the following way (making use of $\left.|\omega|=\left|\omega^{l}\right|+\left|\omega^{g}\right|\right)$

$$
\begin{aligned}
\frac{d}{d t} \int_{\omega^{l}} d V+\int_{\partial \omega^{l} \backslash I} \mathbf{u}^{l} \cdot \eta d S & =0 \\
\int_{\partial \omega} \mathbf{u} \cdot \eta d S & =0,
\end{aligned}
$$


where the first equation is a volumetric contraint on the evolution equation of the interface, and the second equation states that the velocity field is divergence free in integral sense (note that this is not per phase).

\subsection{Velocity discontinuities}

Note that if $\mu^{\pi}=0$ then the velocity field can develop tangential velocity discontinuities $\llbracket u_{\tau} \rrbracket \neq 0$ due to surface tension and/or gravity, where $\tau \perp \eta$ is a vector tangent to the interface. If $\mu^{\pi}>0$ then the NavierStokes solution will be smooth $\llbracket u_{\tau} \rrbracket=0$, numerically we however do not enforce this, as motivated in the introduction.

\section{NUMERICAL MODEL}

The numerical model is implemented in our in-house free surface Navier-Stokes solver ComFLOW which makes use of local and adaptive mesh refinement [11]. For simplicity in presentation however, we will assume the mesh to be 2D and rectilinear. The variables are arranged according to an Arakawa C-grid.

\subsection{Notation}

The notation used is similar to that of [5]. We denote the set of all cells by $C$ and the set of all faces by $\mathcal{F}$, where the faces of one particular cell $c$ are denoted by $\mathcal{F}(c) \subset \mathcal{F}$ and the cells neighbouring one particular face $f$ are denoted by $C(f) \subset C$. Functions $p: c \mapsto p_{c} \approx p\left(\mathbf{x}_{c}\right)$ liv cell c. Similar definition function $\alpha: C \times \mathcal{F} \rightarrow$ pointing relative to the cell $c$. Th

3.2 Interface model
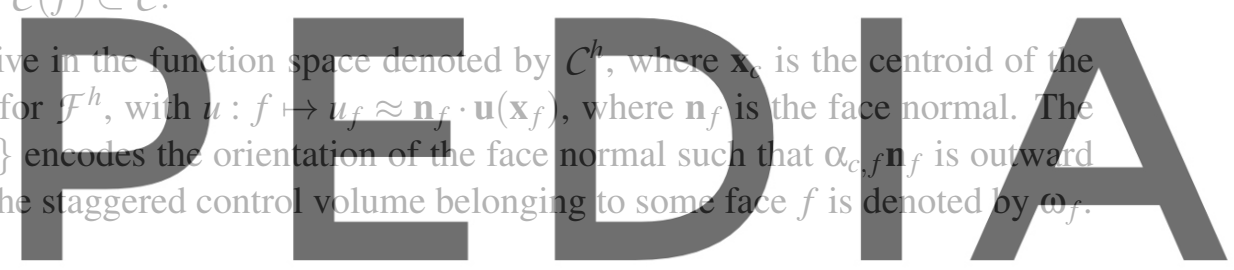

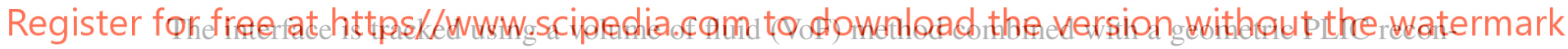
struction.

\subsubsection{Representation}

The interface is represented using the volume fraction field $\bar{\chi}^{l} \in C^{h}$, which is defined as $\bar{\chi}_{c}^{\pi}=\frac{\left|c^{\pi}\right|}{|c|}$. During the interface advection step the volume fluxes are computed using a geometric PLIC reconstruction of the interface: per cell $c$ we find a normal vector $\eta_{c}$ and plane constant $s_{c}$ such that

$$
c^{l}=\left\{\mathbf{x} \in c \mid \eta_{c} \cdot\left(\mathbf{x}-\mathbf{x}_{c}\right) \leq s_{c}\right\}, \quad c^{g}=c \backslash c^{l} .
$$

Computation of the normal vector is done using either local height functions (LHFs) or the efficient leastsquares VoF interface reconstruction algorithm (ELVIRA) [8] if insufficiently many LHFs are available. Using the interface reconstructions we then define the staggered volume fractions $\bar{\chi}^{\pi} \in \mathcal{F}^{h}$ and the phase domains

$$
\bar{\chi}_{f}^{\pi}=\frac{\left|\omega_{f}^{\pi}\right|}{\left|\omega_{f}\right|}, \quad \omega_{f}^{\pi}=\omega \cap \Omega^{\pi}, \quad \Omega^{\pi}=\bigcup_{c \in C} c^{\pi} .
$$




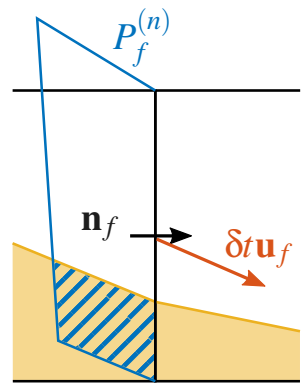

(a) One-fluid model.

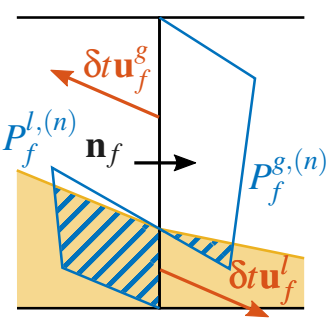

(b) Two-fluid model.

Figure 1: Illustration of the EMFPA flux polygons $P_{f}^{(n)}$ (blue). The blue hatched regions correspond to the liquid volume fluxes and the yellow regions correspond to the liquid domain $\Omega^{l,(n)}$.

The face apertures are given by $a_{f}^{\pi}=\frac{\left|f^{\pi}\right|}{|f|}$, where $f^{\pi}$ is the part of the face $f$ which contains the $\pi$-phase. Note that $f^{\pi}$ is not uniquely defined since the PLIC reconstruction is not continuous accross the faces, therefore we average the face aperture using the two neighbouring cells $c \in C(f)$.

For the modeling of surface tension we compute the interface curvature $\kappa \in C^{h}$ using the LHF method or generalized height function (GHF) method [9] if insufficiently many LHFs are available.

\subsubsection{Advection}

In case of a single fluid we sumn
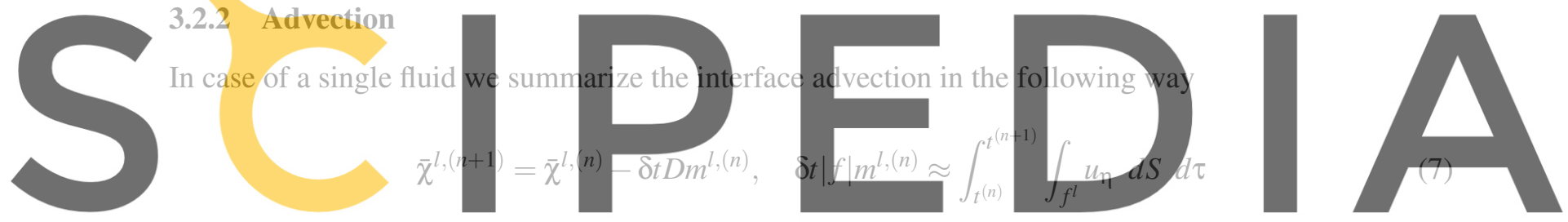

where $D: \mathcal{F}^{h} \rightarrow C^{h}$ is the discrete divergence operator (see section 3.3.5) and $m^{l,(n)}$ denotes the liquid

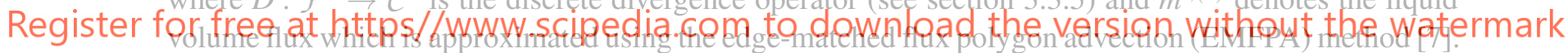

Here the Lagrangian backtracking of the face $f$, resulting in the flux polygon $P_{f}^{(n)}=P^{\operatorname{EMFPA}}\left(f,-\delta t u^{(n)}\right)$, is according to eq. (2), whereas the enforcement step tries to ensure that (here we consider the volume of the flux polygon to be signed)

$$
\left|P_{f}^{(n)}\right|=\delta t|f| u^{(n)} .
$$

The volume flux is then given by

$$
\delta t|f| m_{f}^{l,(n)}=\left|P_{f}^{(n)} \cap \Omega^{l,(n)}\right|,
$$

as shown in fig. 1a.

In case of a velocity discontinuity we modify the original EMFPA method by redefining the flux polygon as

$$
P_{f}^{(n)}=P_{f}^{l,(n)} \cup P_{f}^{g,(n)}, \quad P_{f}^{\pi,(n)}=P^{\mathrm{EMFPA}}\left(f^{\pi,(n)},-\delta t u^{\pi,(n)}\right),
$$

and the volume enforcement step is replaced by

$$
\left|P_{f}^{\pi,(n)}\right|=\delta t|f| a^{\pi,(n)} u^{\pi,(n)} .
$$


See also fig. 1b. It can be shown that this is a consistent approximation to the interface advection problem in the presence of a velocity discontinuity.

\subsection{Momentum equations}

\subsubsection{Convection operator}

The convection operator is very similar to that of [4]. This means that we use volume fluxes from the VoF method to transport momentum, per phase, along with the interface. Our approach differs from that of [4] in a few ways:

- We use the dimensionally unsplit EMFPA method as the underlying advection method. This is mainly because dimensional splitting does not combine well with the fact that our divergence operator depends on the interface configuration (which is updated at each dimensionally split step) via the face apertures (see section 3.3.5).

- In [4] the liquid and gas velocities are 'merged' into a single mass weighted velocity field $\bar{u}$ after each momentum transport step

$$
\bar{u}=\frac{\{\rho \bar{\chi} u\}\}}{\{\rho \rho \bar{\chi}\}\}} .
$$

We skip this step since we allow the velocity field to be discontinuous.

The resulting advection of some function $v^{\pi} \in \mathcal{F}^{h}$ using the velocity field $u^{\pi}$ is denoted by
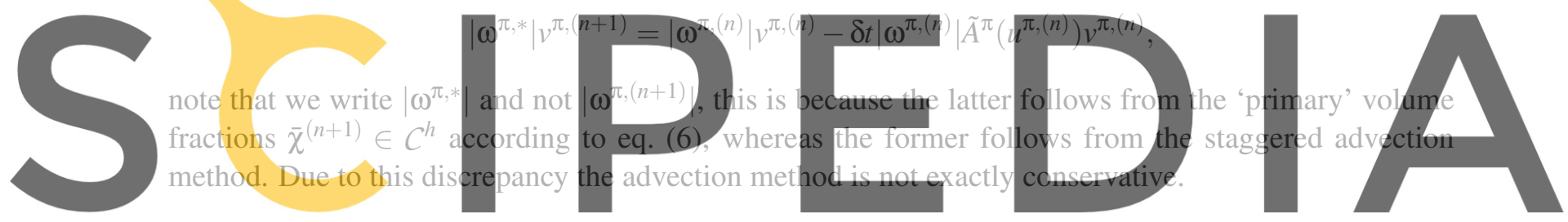

3.3.2 Diffusion operator

Register for free at https//www.scipedia.com to download the version without the watermark

We use a second order accurate diffusion operator applied to $\bar{u}$ with a dynamic viscosity which is based

on weighted harmonic averaging

$$
\bar{\mu}=\left\{\left\{\bar{\chi} \mu^{-1}\right\}^{-1}\right. \text {. }
$$

The reason we use the mass weighted velocity field $\bar{u}$ for diffusion is that we want our solution with a velocity discontinuity to converge to the ordinary Navier-Stokes solution (hence without velocity discontinuity) as soon as the interface layer is resolved $(\delta x \rightarrow 0$ or $\mathrm{Re} \rightarrow 0)$.

\subsubsection{Gravity}

We model gravity according to the following finite volume discretisation

$$
F=\{\{\rho\}\} G\left(\mathbf{g} \cdot \mathbf{x}_{i}\right),
$$

where $\mathbf{x}_{i}$ denotes the interface centroid, and reduces to the cell centroid $\mathbf{x}_{c}$ if the cell does not contain an interface. This model can be shown to sharply mimic the global conservation of energy and is wellbalanced with the gradient operator described in section 3.3.6. That is, if the interface is globally linear, 
then $\exists p \in C^{h}$ for which

$$
F=\{\{\rho \bar{\chi}\}\} g^{\pi}(p) .
$$

\subsubsection{Time integration}

The time integration is split in the following way. First we advect the momentum together with the interface eq. (7)

$$
\left|\omega^{\pi, *}\right| \rho^{\pi} u^{\pi, *}=\left|\omega^{\pi,(n)}\right| \rho^{\pi} u^{\pi,(n)}-\delta t\left|\omega^{\pi,(n)}\right| \tilde{A}^{\pi}\left(u^{\pi,(n)}\right)\left(\rho^{\pi} u^{\pi,(n)}\right) .
$$

Then the diffusion operator and gravity force are integrated in time ${ }^{3}$

$$
u^{\pi, * *}=u^{\pi, *}+\frac{\delta t}{\{\rho \bar{\chi}\}]}\left[\tilde{D}\left(2 \bar{\mu} \tilde{S} \tilde{u}^{*}\right)+F^{(n+1)}\right],
$$

where $\tilde{D}, \tilde{S}$ denote the divergence operator and symmetric part of the gradient operator respectively.

Finally the divergence constraint eq. (5) must be imposed, which is done via a pressure correction step

$$
u^{\pi,(n+1)}=u^{\pi, * *}-\delta t g^{\pi}(p), \quad g^{\pi}(p) \approx \frac{1}{\rho^{\pi}} \mathbf{n}_{f} \cdot\left(\nabla p^{\pi}\right)\left(\mathbf{x}_{f}\right),
$$

for some pressure gradient $g^{\pi}(p)$. We can now also impose the jump condition eq. (3), since this condi-

tion can be written in terms of a jumn condition on the pressure

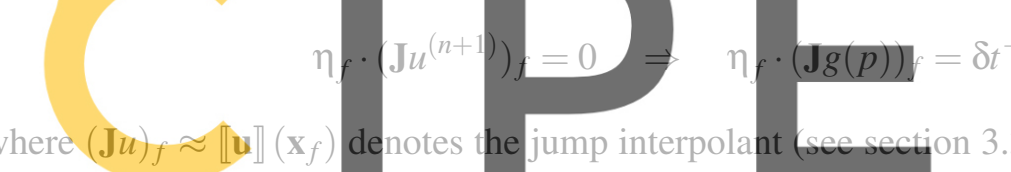

face normal located at $x_{\text {Hence }}$ Hence pressure grad

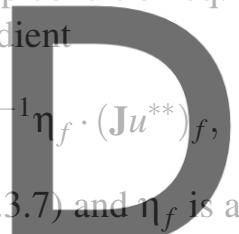

the interface: the Young-Laplace equation eq. (4) as well as the newly introduced pressure oradient jurn

Register fornfirean at. h\&t.ps//www.scipedia.com to download the version without the watermark

\subsubsection{Divergence operator}

The one-fluid divergence operator $D$ is given by

$$
|c|(D u)_{c}=\sum_{f \in \mathcal{F}(c)}|f| \alpha_{c, f} u_{f} \approx \int_{\partial c} \mathbf{u} \cdot \mathbf{n} d S
$$

At the interface two velocity fields exist, and we therefore modify the divergence operator using the cut-cell method (CCM) [10].

$$
|c|\left(D\{\{a u\})_{c} \approx \int_{\partial c^{l} \backslash I} \mathbf{u}^{l} \cdot \mathbf{n} d S+\int_{\partial c^{g} \backslash I} \mathbf{u}^{g} \cdot \mathbf{n} d S=\int_{\partial c} \mathbf{u} \cdot \mathbf{n} d S,\right.
$$

where $|f|\left\{\{a u\}_{f}=\left|f^{l}\right| u_{f}^{l}+\left|f^{g}\right| u_{f}^{g}\right.$ is the total flux through the face $f$, see also fig. 2.

\footnotetext{
${ }^{3}$ For low-Reynolds number flow we use the implicit midpoint rule for the time integration of diffusion.
} 


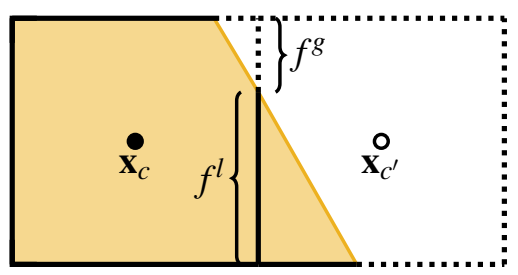

Figure 2: Illustration of the CCM. Each face is split Figure 3: Illustration of the GFM. The pressure valinto its liquid and gaseous part $f=f^{l} \cup f^{g}$. The yellow region corresponds to the liquid domain.

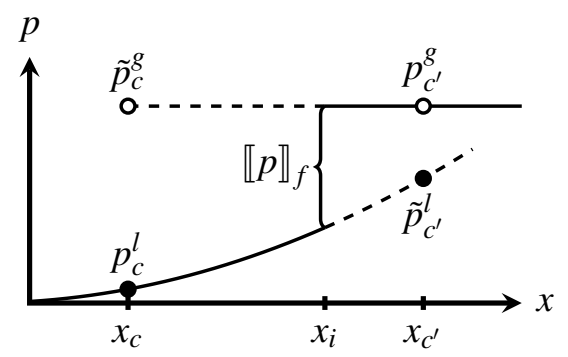

ues denoted by $\tilde{p}$ are 'ghost' pressures and not actually part of the solution. Here $x_{i}=x_{c}+r_{f}^{l}\left(x_{c^{\prime}}-x_{c}\right)$.

\subsubsection{Gradient operator}

In the interior of each of the fluid domains the gradient operator $G$ is simply the negative adjoint of the divergence operator

$$
\left|\omega_{f}\right|(G p)_{f}=-\left|\omega_{f}\right|\left(D^{T} p\right)_{f}=-|f| \sum_{f \in \mathcal{F}(c)} \alpha_{c, f} p_{c} \approx \mathbf{n}_{f} \cdot(\nabla p)\left(\mathbf{x}_{f}\right) .
$$

At the interface we need to modify the gradient to take the two jump conditions into account. Here we use the ghost fluid method (GFM) [6], which results in
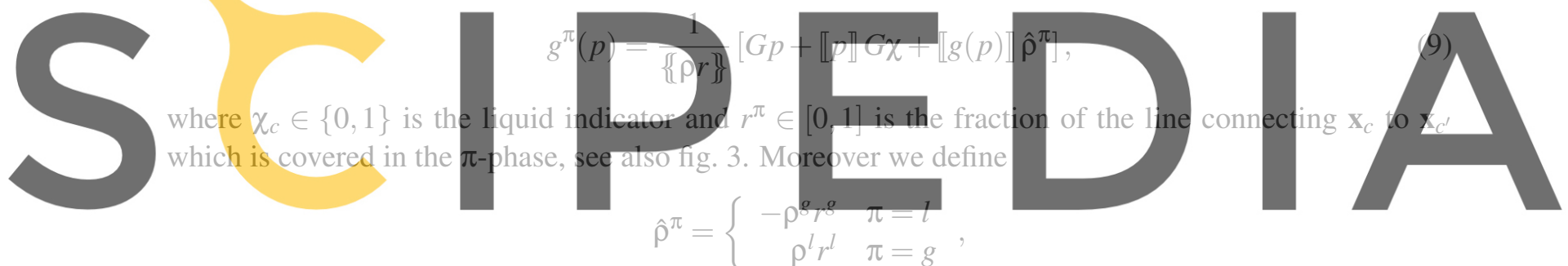

Register for free at https//www.scipedia.com to download the version without the watermark such that $\llbracket \hat{\rho} \rrbracket=\{\rho \rho r\}$ and $\{\rho r \hat{\rho}\}\}=0$. We let $r=\bar{\chi}$ such that momentum is conserved if $\llbracket p \rrbracket=0$

$$
\sum_{f \in \mathcal{F}}\left|\omega_{f}\right| \mathbf{n}_{f}\left\{\{\rho \bar{\chi} g(p)\}_{f}=\sum_{f \in \mathcal{F}}\left|\omega_{f}\right| \mathbf{n}_{f}\left[(G p)_{f}+\llbracket g(p) \rrbracket_{f} \frac{\{\rho \bar{\chi} \hat{\rho}\}_{\}}}{\left\{[\rho \bar{\chi}\}_{f}\right.}\right]=\mathbf{0} .\right.
$$

\subsubsection{The gradient jump}

The GFM eq. (9) requires the face normal component of the gradient jump, $\llbracket g(p) \rrbracket_{f}$, to be known. However, the jump condition eq. (8) specifies the interface normal component of the gradient jump, rather than the face normal component of the gradient jump, which are in general unequal (unless the interface and face normals coincide: $\left.\eta_{f}= \pm \mathbf{n}_{f}\right)$. Hence $\llbracket g(p) \rrbracket_{f}$ is not known a priori, and therefore we include it as an unknown in the pressure Poisson problem, and define it via the imposition of eq. (8).

For imposing eq. (8) we need the jump interpolant $\mathbf{J}$. We let the jump interpolant (in 2D on a rectilinear mesh) be given by

$$
(\mathbf{J} u)_{f}=\mathbf{n}_{f} \llbracket u \rrbracket_{f}+\frac{1}{\left|\mathcal{F}_{I}^{\perp}(f)\right|} \sum_{\hat{f} \in \mathcal{F}_{I}^{\perp}(f)} \mathbf{n}_{\hat{f}} \llbracket u \rrbracket_{\hat{f}},
$$




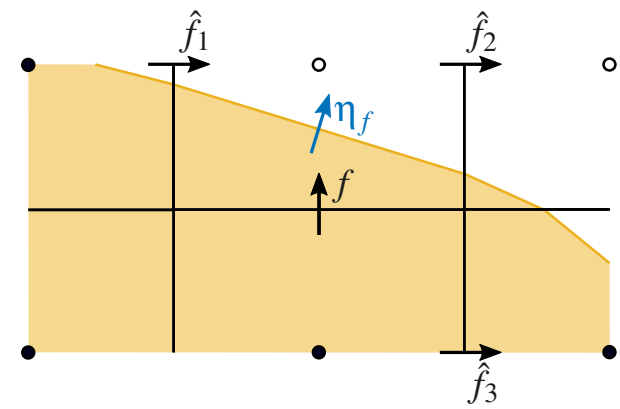

Figure 4: Example jump interpolant for a face $f$ Figure 5: Example jump interpolant for a face $f$ with $\left|\frac{\eta_{f} \cdot \hat{n}_{f}}{\eta_{f} \cdot \mathbf{n}_{f}}\right| \leq 1$. Here $\mathcal{F}_{I}^{\perp}(f)=\left\{\hat{f}_{1}, \hat{f}_{2}, \hat{f}_{3}\right\}$.

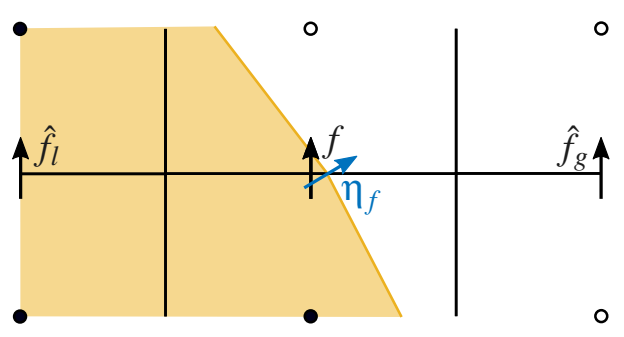
with $\left|\frac{\eta_{f} \cdot \mathbf{n}_{\hat{f}}}{\eta_{f} \cdot \mathbf{n}_{f}}\right|>1$.

where $\mathcal{F}_{I}^{\perp}(f) \subset \mathcal{F}$ contains the faces $\hat{f}$ neighbouring $f$ whose normal $\mathbf{n}_{\hat{f}}$ is perpendicular to $\mathbf{n}_{f}$ and whose corresponding staggered control volume $\omega_{\hat{f}}$ contains both phases, so $\bar{\chi}_{\hat{f}}^{\pi}>0$ for $\pi=\{l, g\}$. Hence the jump in the direction perpendicular to $\mathbf{n}_{f}$ is defined as the average of the available jumps neighbouring the face $f$, see also fig. 4 . We refer to the usage of GFM with our proposed multi dimensional jump interpolant $\mathbf{J}$ as the multi dimensional GFM (MDGFM).

Once eq. (8) is imposed using the previously introduced jump interpolant, we can directly express $\llbracket g(p) \rrbracket_{f}$ in terms of the neighbouring jumps
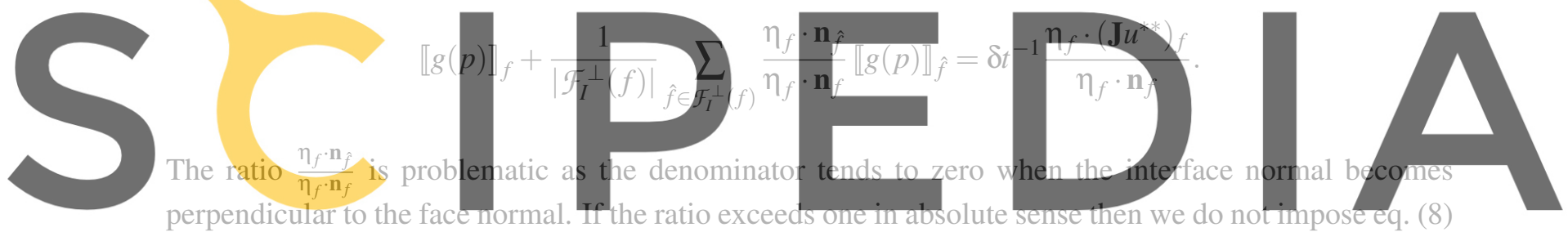

but instead interpolate the jump $\llbracket g(p)$ from neighbouring pressure gradients

Register for free at https//www.scipedia.com to download the version without the watermark

$$
\llbracket g(p) \rrbracket_{f}=g(p)_{\hat{f}_{g}}-g(p)_{\hat{f}_{l}}
$$

where $\hat{f}_{\pi}$ are neighbouring faces whose normal equals that of $f$, as shown in fig. 5 .

The extended pressure Poisson problem can now be written as: find $p \in C^{h}, \llbracket g(p) \rrbracket \in \mathcal{F}_{I}^{h}$ such that

$$
\left\{\begin{array}{rlrl}
D\left\{\{a g(p)\}_{c}\right. & =\delta t^{-1} D\left\{\left\{a u^{* *}\right\}_{c}\right. & & c \in \mathcal{C} \\
\eta_{f} \cdot(\mathbf{J} g(p))_{f} & =\delta t^{-1} \eta_{f} \cdot\left(\mathbf{J} u^{* *}\right)_{f} & & f \in \mathcal{F}_{I} \text { for which }\left|\eta_{f} \cdot \mathbf{n}_{f}\right| \geq \sqrt{\frac{1}{2}} \\
\llbracket g(p) \rrbracket_{f} & =g(p)_{\hat{f}_{g}}-g(p)_{\hat{f}_{l}} & & f \in \mathcal{F}_{I} \text { for which }\left|\eta_{f} \cdot \mathbf{n}_{f}\right|<\sqrt{\frac{1}{2}}
\end{array},\right.
$$

where $\mathcal{F}_{I}$ is the set of faces for which the corresponding staggered control volume contains both phases. Due to the use of the CCM for the divergence operator, it is guaranteed that the right-hand side of the pressure equation is in the image of the Laplace operator, hence a unique (up to a constant) pressure exists. 


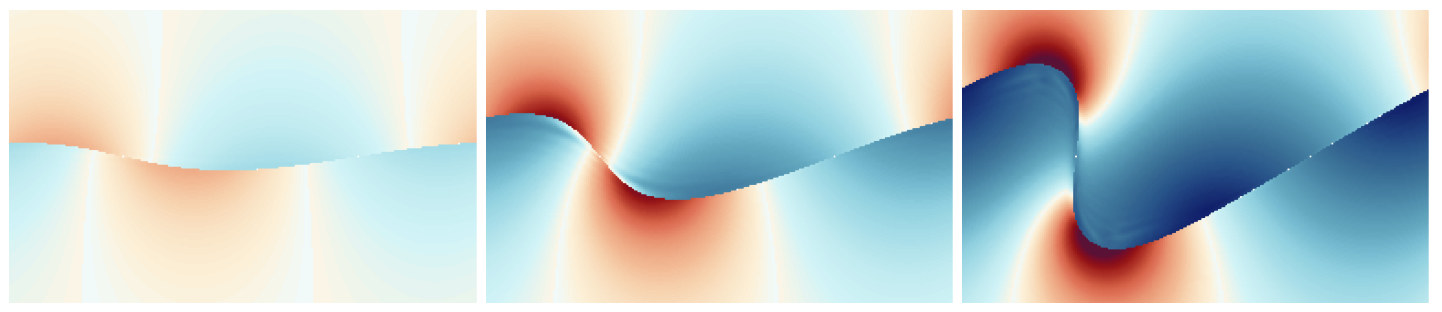

Figure 6: The velocity magnitude (clipped to $|\mathbf{u}|_{2} \in[0,1]$ ) at $t / T^{\sigma}=0.3,0.4,0.5$ for $h / \lambda=1 / 256$.

\section{RESULTS}

\subsection{Inviscid Kelvin-Helmholtz instability}

In order to isolate the treatment of the velocity discontinuity we consider the simulation of an inviscid Kelvin-Helmholtz instability. We let the initial interface profile be given by

$$
\eta(x, 0)=\hat{\eta}(0) \cos (k x), \quad k=\frac{2 \pi}{\lambda}=1, \quad \hat{\eta}(0)=10^{-2},
$$

where the domain, which is periodic in the $x$-direction, is given by $\Omega=[0, \lambda] \times[-1.5 \lambda, 1.5 \lambda]$ with slip boundary conditions applied to the top and bottom walls. The initial velocity field is given by $u^{g}=\delta U / 2$, $u^{l}=-\delta U / 2$ and the corresponding Weber number is given by
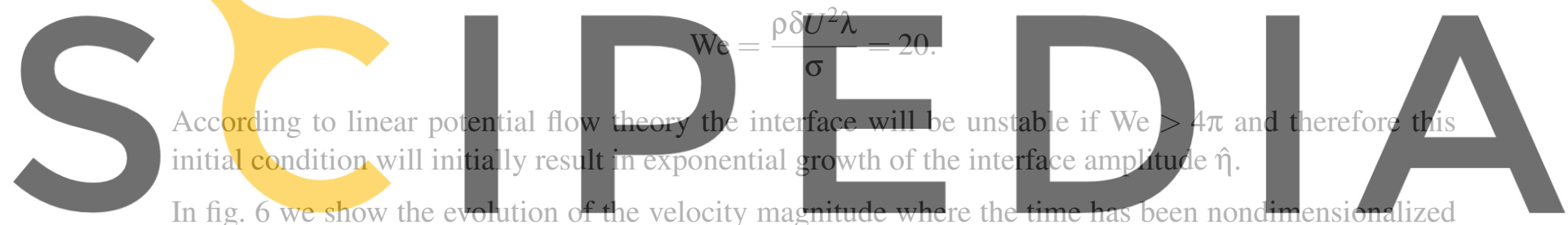

In fig. 6 we show the everution of the vel

using the capillary time scale $T^{\sigma}=\sqrt{\frac{2 p \lambda^{3}}{}}$.

Figures 7 and 8 show convergence to a reference solution which was computed using the author's imple-

mentation of the boundary integral method (BIM) described in [1] (therein refered to as Method III).

\subsection{Third-order Stokes wave}

We consider the simulation of a third-order Stokes wave, for details about the initial conditions and domain size, see [2]. Contrary to [2] we do not initialize the velocity field in the gas phase, hence $u^{g}=0$ at $t=0$. The density ratio is given by $\rho^{g} / \rho^{l} \approx 1.17 \cdot 10^{-3}$. In the notation of [2], we let the Reynolds and Bond number be given by $\mathrm{Re}=4 \cdot 10^{4}$ and $\mathrm{Bo}=10^{3}$ resulting in 'easy to resolve' interface layers and interface length scales. Hence this type of wave is appropriate for testing whether our model will converge to the desired Navier-Stokes solution for a sufficiently fine mesh. For a steepness of $\varepsilon=0.55$ the initial condition results in a plunging breaker. In fig. 9 we show an example simulation using our proposed two-fluid model. Here time has been nondimensionalized in terms of the wave period $T^{g}=\frac{2 \pi}{\sqrt{g k}}$.

In fig. 10 we show the convergence of the interface profile at three time instances as well as the convergence of the tangential velocity jump $\llbracket u_{\tau} \rrbracket$ to zero. This shows that our two-fluid model essentially reduces to the one-fluid model once the interface layer is resolved. 

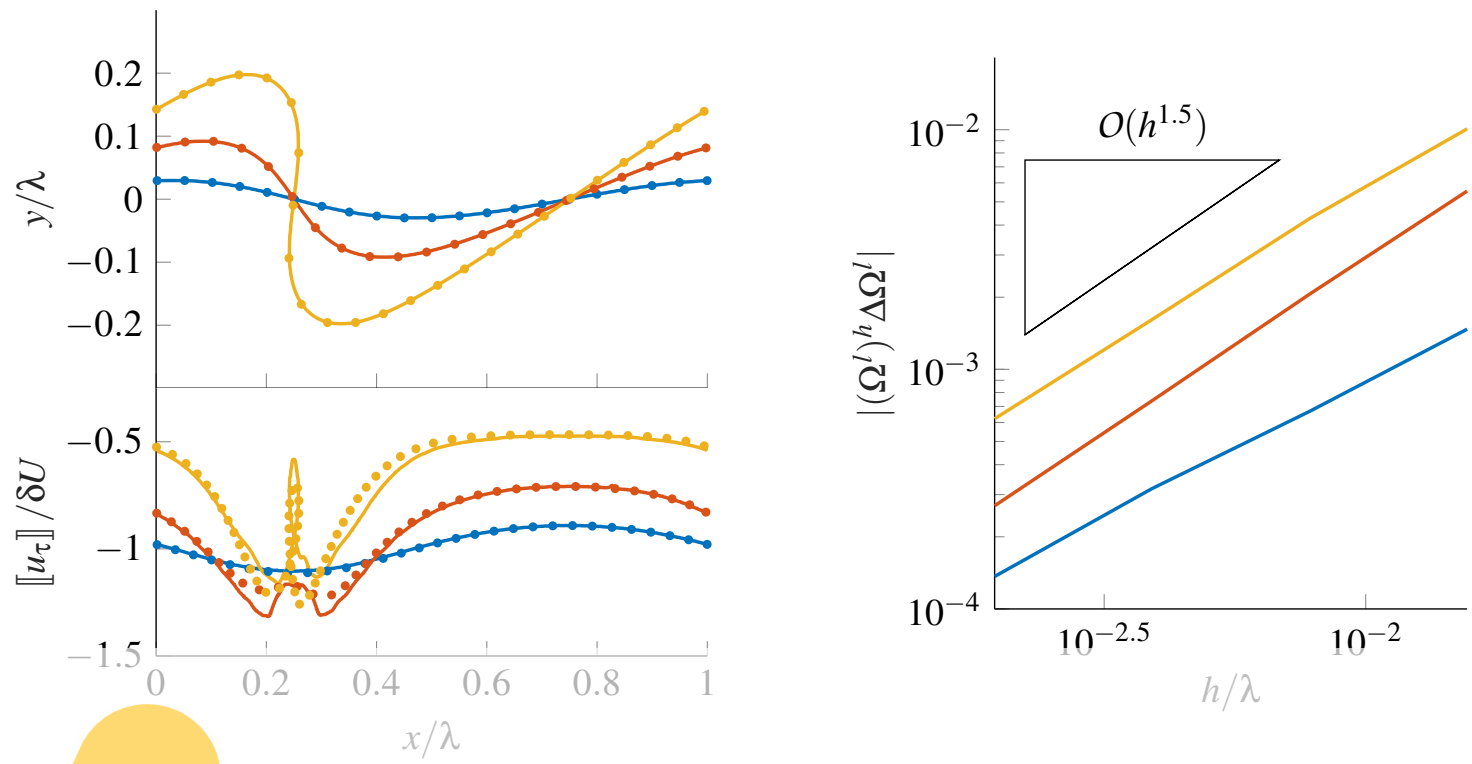

Figure 7: Example of the interface profile (top) and vor- Figure 8: Area of the symmetric differtex sheet strength $\llbracket u_{\tau} \rrbracket$ (bottom) for the Kelvin-Helmholtz ence of the approximate liquid domain problem using $h / \lambda=1 / 512$. The solid lines correspond compared to the BI solution. The blue, to the approximate solution using our proposed method red and yellow lines correspond to $t / T^{\sigma}=$

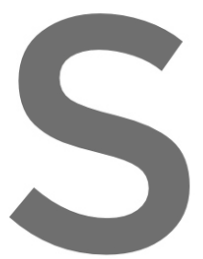
whereas the markers
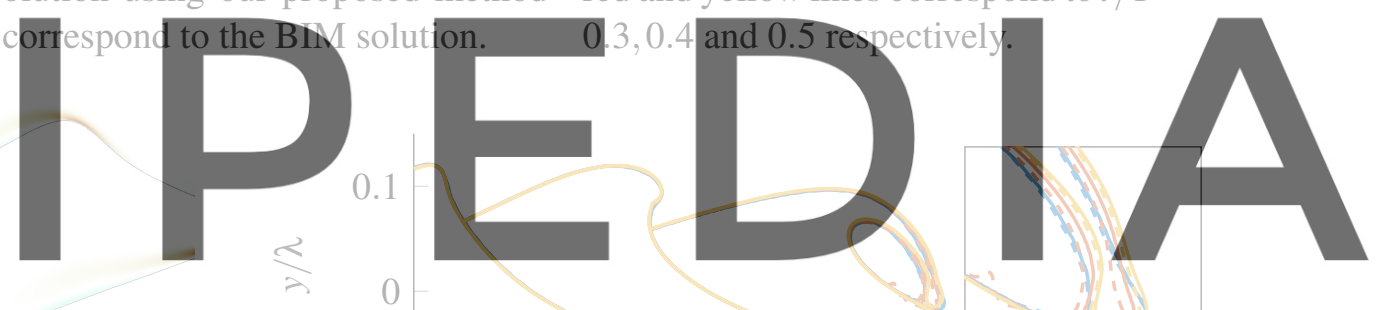

Register for free at https//www.scipedia.com to download the version without the watermark
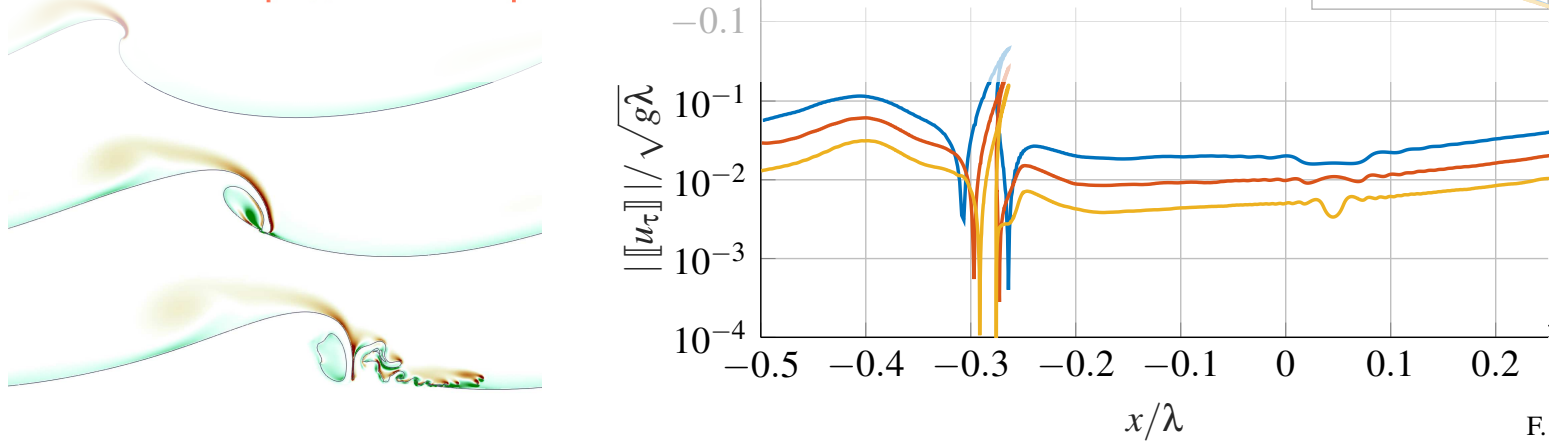

Figure 9: Vorticity (clipped to Figure 10: Convergence of the interface profile (at $t / T^{g} \approx$ $\left.\omega_{y} \in[-100,100]\right)$ and interface $\left.0.36,0.52,0.68\right)$ and tangential velocity discontinuity (at $t / T^{g} \approx$ profile on time instances $t / T^{g} \approx 0.52$ ) for $h / \lambda=2^{-l}$, where $l=9,10,11$ (using AMR) correspond$0.20,0.36, \ldots, 0.84$. Here $h / \lambda=$ $2^{-10}$ (using AMR).

ing to blue, red and yellow respectively. The dashed and solid lines correspond to the one- and two-fluid model respectively. 


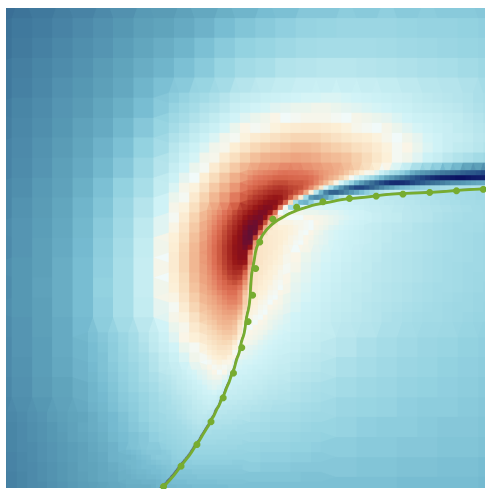

(a) One-fluid model, $l=3$.

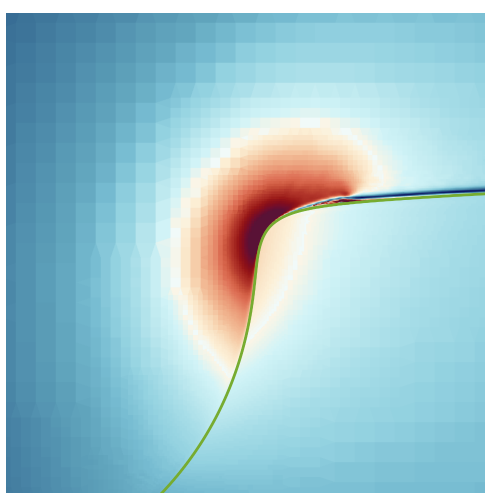

(b) One-fluid model, $l=6$.

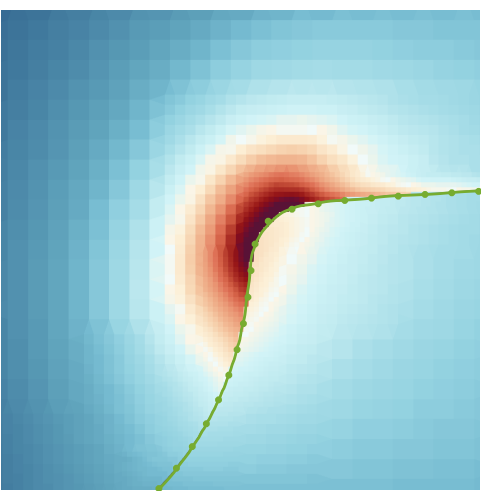

(c) Two-fluid model, $l=3$.

Figure 11: The velocity magnitude (clipped to $|\mathbf{u}| \in[0,15]$ and zoomed in at $[3,6] \times[5,8]$ ) resulting from a large gas-pocket impact at $t=1.62$. The refinement level $l$ refers to an interface resolution of $h=2^{-(2+l)}$. The markers correspond to the interface profile resulting from the one-fluid model with $l=6$.

\subsection{Large gas-pocket impact}

Finally we consider a high Reynolds number test case for which resolving the interface layer is too expensive. We simulate a smoothed dam break which results in a large gas-pocket impact [3]. The fluids (water and air) are initially at rest with the following interface profile

$$
y=7.6+3.6 \tanh (0.44(x-15.5)),
$$

on a domain given by $\Omega=[0,20] \times[0,12] \backslash E$, where $E$ is an ellipse centered at $\mathbf{0}$ with radii given by 18 and 2.8 respectively.

In fig. 11 we show close-ups of the velocity magnitude as well as the interface profile at $t=1.62$ for the one- and two-fluid model ${ }^{4}$. Here we use a resolution of $h=2^{-(2+l)}$ where $l=3$ denotes the maximum level of refinements (we refine at the interface as well as in regions of high vorticity). For reference we also include a more accurate solution resulting from the one-fluid model with $l=6$.

We find that both the one- and two-fluid model yield an accurate interface profile. The one-fluid model however results in an artificially thick interface layer which is not present in the two-fluid model. Further research into how this affects the development of free surface instabilities is however still needed.

\section{DISCUSSION}

We have proposed a sharp two-fluid model for two-phase flow in which underresolved interface layers are modeled by a tangential velocity discontinuity. The numerical model is based on novel generalizations of state-of-the-art numerical methods for the one-fluid model. We demonstrate that the resulting model is able to simulate inviscid (Kelvin-Helmholtz), viscous (Stokes-3 wave) as well as high-Reynolds number (large gas-pocket impact) two-phase flow problems at high accuracy.

\footnotetext{
${ }^{4}$ We picked an early time before impact such that it is still relatively easy to obtain a (nearly) resolved solution.
} 
We will use the proposed model for the simulation of breaking wave impacts and in particular will study the development of free surface instabilities before impact. For future work we suggest the inclusion of compressibility as well as phase-change, the latter of which can sharply be taken into account by including a mass flux through the interface in the CCM divergence operator.

\section{Acknowledgements}

This work is part of the research programme SLING, which is (partly) financed by the Netherlands Organisation for Scientific Research (NWO).

\section{REFERENCES}

[1] G. Baker and A. Nachbin. Stable methods for vortex sheet motion in the presence of surface tension. SIAM Journal on Scientific Computing, 19(5):1737-1766, 1998.

[2] L. Deike, S. Popinet, and K. Melville. Capillary effects on wave breaking. Journal of Fluid Mechanics, 769:541-569, 2015.

[3] S. Etienne, Y.-M. Scolan, and L. Brosset. Numerical Study of Density Ratio Influence on Global Wave Shapes Before Impact. In ASME 2018 37th International Conference on Ocean, Offshore and Arctic Engineering., 2018.

[4] D. Fuster, T. Arrufat, M. Crialesi-Esposito, Y. Ling, L. Malan, S. Pal, R. Scardovelli, G. Tryggvason, and S. Zaleski. A momentum-conserving, consistent, Volume-of-Fluid method for incompressible flow on staggered grids. arXiv preprint:1811.12327, 2018.

[5] K. Lipnikov, G. Manzini, and M. Shashkov. Mimetic finite difference method. Journal of Computational Physics, 257:1163-1227, 2014.

[6] X.-d. Liu, R. P. Fedkiw, and M. Kang. A Boundary Condition Capturing Method for Poisson's Equation on Irregular Domains. Journal of Computational Physics, 160:151-178, 2000.

[7] J. Lopez, J. Hernandez, P. Gomez, and F. Faura. A volume of fluid method based on multidimensional advection and spline interface reconstruction. 195:718-742, 2004.

[8] J. E. Pilliod and E. G. Puckett. Second-order accurate volume-of-fluid algorithms for tracking material interfaces. Journal of Computational Physics, 199(2):465-502, 2004.

[9] S. Popinet. An accurate adaptive solver for surface-tension-driven interfacial flows. Journal of Computational Physics, 228(16):5838-5866, 2009.

[10] H. S. Udaykumar, H.-C. Kan, W. Shyy, and R. Tran-Son-Tay. Multiphase Dynamics in Arbitrary Geometries on Fixed Cartesian Grids. Journal of Computational Physics, 137(2):366-405, 1997.

[11] P. Van der Plas. Local grid refinement for free-surface flow simulations. PhD thesis, Rijksuniversiteit Groningen, 2017. 